\title{
EFFECTS OF OVARY STORAGE TIME ON THE QUALITY AND MEIOTIC COMPETENCE OF CAT OOCYTES
}

\author{
V.V. Luu, Z. Namula, Y. Kaedei, F. Tanihara and T. Otoi \\ Laboratory of Animal Reproduction, the United Graduate School of Veterinary Science, \\ Yamaguchi University, Yamaguchi, Japan
}

Unlike the oocytes of other species, cat oocytes have a unique characteristic in that they can mature in vitro after temporary storage at $5^{\circ} \mathrm{C}$. Temporal storage of ovaries can aid in preserving oocytes from the ovaries of endangered felids that sometimes die suddenly in the field. The present study was conducted to evaluate the effects of the duration of cold storage of cat ovaries on the quality and meiotic competence of their oocytes. Domestic cat ovaries were collected from sexually mature queens (40 ovary pairs) at various stages of the reproductive cycle, after routine ovariohysterectomy performed at local veterinary clinics. After excision, the ovaries were stored at $5^{\circ} \mathrm{C}$ in physiological saline for $0,1,2,3,4$, and 5 days until oocyte recovery. The cumulus-oocyte complexes with uniform and dark pigmented ooplasm and more than 2 layers of cumulus cells were collected $(n=526)$ and then cultured for $24 \mathrm{~h}$ in maturation medium. This maturation medium consisted of tissue culture medium 199 supplemented with $4 \mathrm{mg} \mathrm{mL}^{\wedge}\{-1\}^{\wedge}$ bovine serum albumin, $0.1 \mathrm{IU} \mathrm{mL}^{\wedge}\{-1\}^{\wedge}$ human menopausal gonadotropin, $10 \mathrm{IU} \mathrm{mL}^{\wedge}\{-1\}^{\wedge}$ human chorionic gonadotropin, $1 \mu \mathrm{g} \mathrm{mL} \mathrm{m}^{\wedge}\{-1\}^{\wedge}$

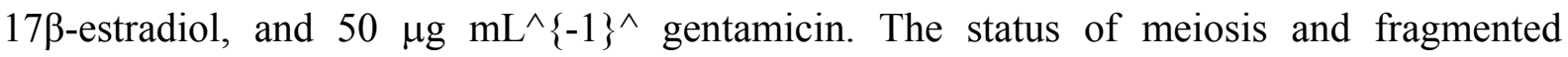
chromatin in the oocytes after $24 \mathrm{~h}$ of maturation culture was assessed by terminal deoxynucleotidyl transferase dUTP nick end labeling and Hoechst 33342 staining. Data were analyzed using analysis of variance. The percentage of oocytes that reached metaphase II was greater for ovaries kept without cold storage (control group; 60.5\%; $P<0.05$ ) than for ovaries kept for 1, 2, 3, 4 and 5 days in cold storage (maturation of $28.6 \%, 38.3 \%, 23.2 \%, 1.6 \%$ and $2.2 \%$, respectively, $\mathrm{P}<0.05)$. In the cold storage group, the maturation rates $(1.6-2.2 \%)$ of oocytes from ovaries stored for more than 4 days were significantly lower $(P<0.05)$ than those (23.2-38.3\%) of oocytes from ovaries stored for less than 3 days. Moreover, oocytes with fragmented chromosomes after maturation culture were not observed in the case of the control group, whereas $24.4-51.8 \%$ of the oocytes in the cold storage group had fragmented chromosomes, irrespective of the storage period. These results indicate that cold storage of cat ovaries decreases the meiotic competence of oocytes and influences the quality of oocytes matured in vitro. 